OPEN ACCESS

Edited by:

John Perfect,

Duke University, United States

Reviewed by:

Frederic Lamoth,

Centre Hospitalier Universitaire

Vaudois (CHUV), Switzerland

Taissa Vila

Federal University of Rio de Janeiro,

Brazil

${ }^{*}$ Correspondence: Mahmoud A. Ghannoum

mag3@case.edu

${ }^{\dagger}$ These authors have contributed equally to this work and share first

authorship

Specialty section:

This article was submitted to

Fungal Pathogenesis,

a section of the journal

Frontiers in Cellular and

Infection Microbiology

Received: 15 December 2020 Accepted: 05 February 2021

Published: 11 March 2021

Citation:

Gamal A, Chu S, McCormick TS,

Borroto-Esoda K, Angulo D and Ghannoum MA (2021) Ibrexafungerp,

a Novel Oral Triterpenoid Antifungal in

Development: Overview of Antifungal

Activity Against Candida glabrata.

Front. Cell. Infect. Microbiol. 11:642358.

do: 10.3389/fcimb.2021.642358

\section{Ibrexafungerp, a Novel Oral Triterpenoid Antifungal in Development: Overview of Antifungal Activity Against Candida glabrata}

\author{
Ahmed Gamal ${ }^{1+}$, Sherman Chu ${ }^{1,2 \dagger}$, Thomas S. McCormick ${ }^{1}$, Katyna Borroto-Esoda ${ }^{3}$, \\ David Angulo ${ }^{3}$ and Mahmoud A. Ghannoum ${ }^{1,4 *}$ \\ ${ }^{1}$ Department of Dermatology, Case Western Reserve University, Cleveland, OH, United States, ${ }^{2}$ College of Osteopathic \\ Medicine of the Pacific, Northwest (COMP), Lebanon, OR, United States, ${ }^{3}$ Scynexis Inc., Jersey City, NJ, United States, \\ ${ }^{4}$ Department of Dermatology, Center for Medical Mycology, University Hospitals Cleveland Medical Center, Cleveland, \\ $\mathrm{OH}$, United States
}

Systemic infections caused by Candida species are an important cause of morbidity and mortality among immunocompromised and non-immunocompromised patients. In particular, Candida glabrata is an emerging species within the Candida family that causes infections ranging from superficial to life-threatening systemic disease. Echinocandins and azoles are typically the first-line therapies used to treat infections caused by C. glabrata, however, there is an increasing prevalence of resistance to these antifungal agents in patients. Thus, a need exists for novel therapies that demonstrate high efficacy against $C$. glabrata. Ibrexafungerp is a first-in-class glucan synthase inhibitor with oral availability developed to address this increasing antifungal resistance. Ibrexafungerp demonstrates broad in vitro activity against wild-type, azole-resistant, and echinocandinresistant $C$. glabrata species. Furthermore, ibrexafungerp has shown efficacy in low pH environments, which suggests its potential effectiveness in treating vulvovaginal candidiasis. Additional preclinical and clinical studies are needed to further examine the mechanism(s) of ibrexafungerp, including acting as a promising new agent for treating $C$. glabrata infections.

Keywords: ibrexafungerp, Candida glabrata, B-(1,3)-D-glucan, triterpenoid class (fungerps), antifungal

\section{INTRODUCTION}

The incidence of fungal infections has increased significantly over the last several decades and are important causes of morbidity and mortality (Lass-Florl, 2009). Candida species are the most common cause of invasive fungal infections and are associated with high mortality and morbidity rates among both immunocompromised and non-immunocompromised hosts (Dodds Ashley et al., 2012; Pfaller M. et al., 2012). Although Candida albicans is the most prevalent Candida species isolated from humans, Candida glabrata infections have increased significantly over the last two decades and are now one of the primary causes of candidemia in the United States, accounting for more than a third of all candidemia isolates (Bassetti et al., 2006; Chakrabarti et al., 2009; 
Lockhart et al., 2012; Matsumoto et al., 2014). The reason for the increasing trend of C. glabrata infections is multifactorial and can be due to geographical variation, differing patient populations, and increased use of prophylactic fluconazole (Richardson and Lass-Florl, 2008).

In addition to systemic infections, superficial infections caused by Candida are also important.

In particular, vulvovaginal candidiasis (VVC) is the second most common cause of vaginitis after bacterial vaginosis (Anderson et al., 2004). The incidence of VVC is substantial, with estimations of up to $70-75 \%$ of women experiencing an episode of VVC and it has been estimated that approximately $10-15 \%$ of asymptomatic women are colonized with a Candida species (Sobel, 2007). C. glabrata is the second most common species identified in women with VVC after C. albicans with a prevalence ranging between 3.4 to $20 \%$ in America, Europe, and Australia (Corsello et al., 2003; Holland et al., 2003; Sojakova et al., 2004; Richter et al., 2005; Grigoriou et al., 2006; Paulitsch et al., 2006). Several studies have reported higher prevalence of $C$. glabrata causing VVC (29.5-50.4\%), specifically in studies performed in Asian and African countries (Okungbowa et al., 2003; Cetin et al., 2007; Mohanty et al., 2007; Ahmad and Khan, 2009). Additionally, older women, women with uncontrolled diabetes, and women afflicted with HIV have been associated with high percentages of C. glabrata species causing VVC (Goswami et al., 2000; Corsello et al., 2003; Dan et al., 2006; Fan et al., 2008). Published studies suggested that C. glabrata infections emerge as breakthrough infections in women following a maintenance low-dose fluconazole prophylaxis. It is believed that recurrent vulvovaginal candidiasis has a mutual relationship with non-albicans Candida infections, where the occurrence of one predisposes to others. Moreover, recurrent infections result in increased frequency of non-albicans Candida colonization, while the persistence of these non-albicans Candida can also lead to recurrent infections (Fidel et al., 1999; Makanjuola et al., 2018).

\section{RESISTANCE}

Despite C. glabrata lacking several virulence factors associated with other Candida species, such as hyphal growth and the ability to secrete proteases, this species is a growing concern for mucosal and systemic bloodstream infections (Kaur et al., 2005; Lim et al., 2012; Sardi et al., 2013). An active population-based surveillance for culture-confirmed candidemia was conducted by the Centers for Disease Control and Prevention (CDC) during 2012-2016 in four U.S. states (Georgia, Maryland, Oregon, and Tennessee). Out of 3,492 cases of candidemia, C. glabrata accounted for the second highest organism detected, resulting in $28 \%$ of cases compared to C. albicans that accounted for $38 \%$ (Toda et al., 2019). A major concern is that C. glabrata resistance to currently available antifungals is increasing and the emergence of multidrug resistance threatens the effectiveness of both azoles and echinocandins (Pfaller and Diekema, 2007). In general, resistance to antifungal agents may be either intrinsic, such as the resistance of $C$. krusei to fluconazole, or acquired-as a result of extensive use of antifungal agents (Arendrup and Patterson, 2017).

C. glabrata has been found to have higher rates of azole antifungal resistance in comparison to other Candida species, such as C. albicans, C. tropicalis, and C. parapsilosis (Lockhart et al., 2012). In a study published in 2012, two hundred ninetyone (291) C. glabrata isolates from around the world demonstrated low susceptibility when tested against a panel of antifungal agents including triazoles (Castanheira et al., 2014). This may be due to the widespread use of azoles, especially in patients using these agents for long term prophylaxis and treatment. This, in turn, results in the emergence of intrinsically less susceptible strains of C. glabrata (Redding et al., 2004). Various mechanisms of resistance to azoles have been described in the literature including gene mutation (ERG11 mutation causing less binding), gain of function mutation (GOF), gene up-regulation (GOF in UPC2 leading to overexpression of ERG11 causing insufficient azole activity), and overexpression of efflux pumps (decreasing the intracellular concentrations of the drug) (Sanglard, 2016). In this regard, $C g C D R 1$ and $C g C D R 2$ transporters are found to be the main mechanism for azole resistance in C. glabrata which occur as a result of CgPDR1 GOF mutation (Ghannoum and Rice, 1999; Borst et al., 2005; Vale-Silva et al., 2013; Sanglard and Coste, 2016).

Additionally, C. glabrata resistance to echinocandins is increasingly prevalent with rates ranging between 3 and $30 \%$ (Alexander et al., 2013). This finding is particularly noted in patients who are extensively using echinocandins, especially in strains exhibiting less susceptibility to fluconazole and other azoles (Beyda et al., 2014). Resistance to echinocandins has only been linked to mutations in two hot spot regions of the FKS genes leading to amino acid substitutions in these regions (hot spots 1 and 2 of FKS1 gene for C. albicans and hot spot 1 and 2 of FKS1 and FKS2 gene for C. glabrata) (Sanglard, 2016). Echinocandins susceptibility test was evaluated against a total of 1,380 isolates of C. glabrata collected between 2008 and 2013 from four U.S. cities. Screening for FKS mutations was done in 1,032 isolates including 77 isolates showing intermediate or resistant MIC values to at least one echinocandin. A total of 51 isolates were found to have FKS mutations, 15 isolates with FKS1 mutations and 35 with FKS2 mutations. It was also found that not all FKS mutations cause the same degree of resistance which is demonstrated by the variable MICs among these isolates (Pham et al., 2014). Within FKS1, mutations at S629 caused higher MICs values than others occurring at R631 or D632. Furthermore, mutations at R631 were associated with higher micafungin MICs but lower values for caspofungin and anidulafungin. Additionally, D632V mutations demonstrated elevated MIC values to all echinocandins, which was not the case for the D632Y mutation where high MIC values were not reported for any of the echinocandins tested.

A major concern is the potential development of multidrug resistant (MDR) C. glabrata strains. In a study based on the SENTRY Antimicrobial Surveillance Program for the years 2006 
through 2010 and the Centers for Disease Control and Prevention population-based surveillance conducted in 20082010, out of 1,669 C. glabrata isolates, 162 were resistant to fluconazole including 18 isolates that were also resistant to one or more of the echinocandins. In all 18 isolates, mutations in either FKS1 or FKS2 were detected (Pfaller M. A. et al., 2012). Furthermore, in a population-based active surveillance for culture-confirmed candidemia in four sites in the U.S. during 2012-2016, out of 929 C. glabrata isolates, 12 isolates were MDR (Toda et al., 2019).

In addition to resistance mechanisms described previously, a newly proposed mechanism that may explain the rapid acquisition of fluconazole, echinocandin, and amphotericin B resistance is $\mathrm{MSH} 2$ DNA mismatch repair gene mutations. Out of 357 geographically diverse C. glabrata strains, these mutations were detected in nearly half of the isolates (susceptible and resistant). Interestingly, these mutations were more frequent among fluconazole-resistant and MDR C. glabrata isolates (Healey et al., 2016).

The increasing resistance to azoles and echinocandins in addition to amphotericin B toxicity and lack of oral bioavailability of these drugs calls for the development of new antifungals. Additional compelling support for the development of new effective antifungal to combat infection caused by C. glabrata is the recent report of amphotericin B resistant C. glabrata strains (Farmakiotis et al., 2014; Cho et al., 2015).

\section{IBREXAFUNGERP}

\section{Mechanism of Action}

Ibrexafungerp (formerly SCY-078) is the first compound of the enfumafungin-derived triterpenoid class of antifungals and the first oral (1-3)- $\beta$-D-glucan synthase inhibitor (GSIs) (Ghannoum et al., 2020a). GSIs are a group of drugs that act by inhibition of $\beta$-1,3-D-glucan synthase, a key enzyme in the biosynthesis of $B-(1,3)$-D-glucan, a major component of the fungal cell wall.

$\beta$-1,3-D-glucan synthase is unique to lower eukaryotes thus, antifungal agents that share this fungal-specific mode of action tend to exhibit low toxicity. GSIs were first introduced in 2001, with caspofungin the first echinocandin to be approved. Two additional echinocandins were later introduced, micafungin and anidulafungin (Heasley et al., 2012). The main advantages of echinocandins are broad-spectrum and fungicidal activity with a safety profile better than other antifungal agents. However, the key limitation of the echinocandins is the requirement for administration by intravenous infusion hence there is a need for new agents that can be administered orally with the preservation of these features (Walker et al., 2011).

Natural compounds screening led to the discovery of enfumafungin, a triterpene glycoside natural product, which was found to have in vitro antifungal activity comparable to caspofungin. However, it showed limited stability in vivo. Derivatives of enfumafungin were found to be potent inhibitors of $\beta$-1,3-D-glucan synthase and are structurally different from the echinocandins. Semi-synthetic modification of these derivatives resulted in improvement of oral bioavailability and PK properties thereby leading to the discovery of ibrexafungerp (Ghannoum et al., 2020a).

The aim of ibrexafungerp development is to be the first oral and IV GSI that can be used in the treatment and prevention of various fungal infections including life-threatening infections (Davis et al., 2020) as well as superficial ones (e.g., vulvovaginal candidiasis). Ibrexafungerp and echinocandins act by inhibition of $\beta$-1, 3-D-glucan synthase; however, they are different in structure and interact differently with the target enzyme resulting in a lower rate of resistance to ibrexafungerp (Jimenez-Ortigosa et al., 2017). Furthermore, the binding site for both appears to be nonidentical. In a recent study, ibrexafungerp demonstrated high activity against Candida species including wild-type and echinocandin-resistant strains in the presence of FKS mutations suggesting limited potential for cross-resistance with echinocandins (Pfaller et al., 2017). However, the identification of the binding site is currently under investigation.

\section{In Vitro and In Vivo Activity}

Ibrexafungerp displayed potent in vitro activity against a wide range of Aspergillus species and Candida isolates, including isolates resistant to azoles and echinocandins (JimenezOrtigosa et al., 2014; Ghannoum et al., 2018). It also showed consistent activity against $C$. glabrata with $f k s$ mutations, which confer resistance to echinocandins, with MIC values ranging from $<0.03$ to $4 \mu \mathrm{g} / \mathrm{ml}$ (Nunnally et al., 2019). Additionally, ibrexafungerp has uniform and potent activity against C. auris, an emerging multidrug-resistant fungus, which was demonstrated in several studies (Berkow et al., 2017; Arendrup et al., 2020; Barat SB-E, 2020; Ghannoum et al., 2020a). Furthermore, ibrexafungerp has also shown the ability to reduce $C$. auris tissue fungal burden in an in vivo guinea pig model (Ghannoum et al., 2020b).

Interestingly, studies showed that ibrexafungerp inhibited cell growth and division of $C$. auris. These data suggest that in addition to the glucan synthase inhibition effect, this drug may have a separate target or may affect this enzyme through different mechanisms (Larkin et al., 2017). Ibrexafungerp has fungicidal activity against Candida species which was demonstrated in a time-kill study in which ibrexafungerp showed activity comparable to that of caspofungin. It also showed potent in vitro activity against Candida species in a low $\mathrm{pH}$ environment suggesting a therapeutic advantage of ibrexafungerp in the treatment of vaginal Candida infections (Larkin et al., 2019).

\section{Pharmacokinetics}

Several studies were done to determine pharmacokinetic parameters and bioavailability of ibrexafungerp. In vitro bioavailability was assessed using Caco-2 cell monolayers as a predictor of absorption across the gut. The mean apparent permeability was $8.9 \pm 0.78 \times 10^{-6} \mathrm{~cm} / \mathrm{s}$ for $5 \mu \mathrm{M}$ ibrexafungerp indicating a good oral absorption. This was consistent with the data obtained following oral administration in animal models which displayed an oral bioavailability of $35 \%$ $50 \%$ (calculated by oral $\mathrm{AUC}_{0-12}$ versus intravenous $\mathrm{AUC}_{0-\infty}$ ) (Wring et al., 2017). It also demonstrated high protein binding 
(99.5-99.8\%) in different species, which is expected for a compound with a lipophilic nature.

Ibrexafungerp achieved maximum plasma concentrations $\left(\mathrm{C}_{\max }\right)$ between 4 and $6 \mathrm{~h}$ reflecting a prolonged absorption phase after oral administration. In animals, observation of drug levels after a single dose showed a monophasic decline with a half-life of 7-9 h (Lepak et al., 2015; Wring et al., 2019a). A Phase 1 study in healthy volunteers demonstrated peak plasma concentration within 4-6 h with a linear decline and a mean terminal half-life of approximately $20-30 \mathrm{~h}$. In the same setting, administration of ibrexafungerp with a high-fat meal showed increased bioavailability, on the other hand, food delayed the absorption of ibrexafungerp from a median time to peak concentration (Tmax) of $4.0 \mathrm{~h}$ in the fasted state to $6.0 \mathrm{~h}$ in the fed state (Trucksis et al., 2009).

In animals, ibrexafungerp displayed wide tissue distribution with a steady-state volume of distribution (Vdss) of $>5$ liter $/ \mathrm{kg}$ (Wring et al., 2019a), which is several-fold greater than fluconazole and echinocandins (Lepak et al., 2015). Furthermore, assessment of the distribution of ibrexafungerp between plasma and kidney tissue displayed a tissue concentration 20- to 25-fold greater than that seen in plasma, indicating high tissue penetration. In comparison with micafungin, the mainstay in the treatment of intra-abdominal candidiasis, ibrexafungerp demonstrated superior lesion penetration properties in mice with intra-abdominal candidiasis (Lee et al., 2020). After a single oral dose of ibrexafungerp $(15 \mathrm{mg} / \mathrm{kg})$ in rats, ratios of tissue-to-blood AUC in commonly affected organs by invasive fungal disease were: spleen 54-fold; liver 50-fold; lung 31-fold; bone marrow 25 fold; kidney 20-fold; skin 12-18-fold; vaginal tissue 9-fold; and skeletal muscle 4-fold, with minimal distribution to central nervous system tissues (Wring et al., 2019a). Ibrexafungerp showed a high potential to accumulate in vaginal tissue and fluids with a tissue concentration 2-5 folds higher than plasma concentration. Carbon labeled $\left[{ }^{14} \mathrm{C}\right]$ ibrexafungerp was used to determine the elimination route for ibrexafungerp in animal models following intravenous (I.V.) and oral administration.

Most of the radioactivity $(\sim 80 \%)$ was recovered from feces for both routes while nearly $1 \%$ was recovered from urine. Total recovery of the radioactivity of the administered I.V. and oral doses was 92.2 and 91.8\% respectively (Larkin et al., 2019).

Safety of ibrexafungerp was evaluated in a total of 12 subjects that received a loading dose of 1250-mg ibrexafungerp on day 1 followed by daily maintenance doses of 750-mg SCY 780 citrate on days 2 and 3. Blood samples were collected predose and postdose on both days 1 and 3. Adverse events (AE) were reported in 6 subjects that were mostly in the form of diarrhea, abdominal pain, nausea, vomiting, and flatulence. However, these $\mathrm{AE}$ were mild or moderate in intensity with no serious $\mathrm{AE}$ reported and no subjects discontinued the study due to an $\mathrm{AE}$ (Wring et al., 2019b).

There was no clinically relevant effect on the QTc interval in healthy volunteers at a plasma concentration up to $4 \mu \mathrm{g} / \mathrm{ml}$ (Murphy et al., 2017). Ibrexafungerp also did not affect fertility or early embryonic development of male and female rats using doses above clinically efficacious doses which suggest potential use in the treatment of fungal infection in women during pregnancy (Carruthers et al., 2018).

In vitro studies demonstrated that ibrexafungerp is a CYP3A4 substrate and reversible inhibitor of CYP2C8 and CYP3A4. However, coadministration of a single-dose of ibrexafungerp with rosiglitazone (CYP2C8 substrate), and tacrolimus (CYP3A4 substrate) showed minimal effect on the pharmacokinetics of both drugs, suggesting low potential for ibrexafungerp to cause CYP-mediated drug interactions at therapeutic exposures. Moreover, data showed that co-administration of a single dose of tacrolimus with ibrexafungerp at a steady-state has no effect on the maximum blood level of tacrolimus with only a 1.4-fold increase in systemic exposure to tacrolimus which is markedly weaker interaction than observed with azoles. These findings are supportive for coadministration of ibrexafungerp and tacrolimus without the need for initial dose adjustment for tacrolimus (Wring et al., 2018; Wring et al., 2019b).

\section{Clinical Development Overview}

Four clinical trials, CANDLE-304, SCYNERGIA, CARES, and FURI, are ongoing for further evaluation of ibrexafungerp. CANDLE-304 is a phase 3 study to evaluate the efficacy and safety of oral ibrexafungerp in patients with recurrent vulvovaginal candidiasis (VVC) ([[NoAuthor]]), SCYNERGIA is a multicenter randomized double-blind study to evaluate the safety and efficacy of coadministration of ibrexafungerp with voriconazole in patients with invasive pulmonary aspergillosis (A Multicenter, Randomized, Double-Blind Study to Evaluate the Safety and Efficacy of the Coadministration of SCY-078 With Voriconazole in Patients With Invasive Pulmonary Aspergillosis [Internet]), CARES is an open-label study to evaluate oral ibrexafungerp as an emergency use treatment for patients with candidiasis including C. auris candidemia (Open-Label Study to Evaluate the Efficacy, Safety, Tolerability and Pharmacokinetics of Oral Ibrexafungerp (SCY-078) as an Emergency Use Treatment for Patients With Candidiasis, Including Candidemia, Caused by Candida Auris [Internet]) and FURI is an open-label study to evaluate the efficacy and safety of ibrexafungerp in patients with fungal diseases refractory to standard treatment (Open-Label Study to Evaluate the Efficacy and Safety of SCY-078 (Ibrexafungerp) in Patients With Fungal Diseases That Are Refractory to or Intolerant of Standard Antifungal Treatment (FURI) [Internet]).

\section{IBREXAFUNGERP AND CANDIDA GLABRATA}

\section{In Vitro Activity}

Ibrexafungerp has been tested in vitro against C. glabrata isolates with FKS1 and FKS2 mutations, which have varying degrees of susceptibility to echinocandins. The in vitro activity of ibrexafungerp was determined for 34 C. glabrata clinical isolates with genotypic, phenotypic, or clinical echinocandin resistance (Schell et al., 2017). The MIC $_{90}$ (minimum 
concentration that inhibited $90 \%$ of tested isolates) for ibrexafungerp against resistant C. glabrata was $4 \mu \mathrm{g} / \mathrm{ml}$, while fluconazole and caspofungin showed $\mathrm{MIC}_{90}$ values of 128 and 8 $\mu \mathrm{g} / \mathrm{ml}$, respectively. Anidulafungin and micafungin exhibited $\mathrm{MIC}_{90}$ values of $2 \mu \mathrm{g} / \mathrm{ml}$ for both drugs against C. glabrata, while voriconazole showed $\mathrm{MIC}_{90}$ values of $4 \mu \mathrm{g} / \mathrm{ml}$. In $23 \mathrm{C}$. glabrata clinical isolates from episodes of bloodstream infections, $\mathrm{MIC}_{90}$ for ibrexafungerp was $1 \mu \mathrm{g} / \mathrm{ml}$ with fluconazole demonstrating $\mathrm{MIC}_{90}$ values of $64 \mu \mathrm{g} / \mathrm{ml}$ (Schell et al., 2017).

In 89 echinocandin resistant C. glabrata isolates, MIC values of ibrexafungerp ranged from $<0.03 \mu \mathrm{g} / \mathrm{ml}$ to $4 \mu \mathrm{g} / \mathrm{ml}$ with $\mathrm{MIC}_{50}$ (minimum concentration that inhibited $50 \%$ of tested isolates) and $\mathrm{MIC}_{90}$ values of $0.25 \mu \mathrm{g} / \mathrm{ml}$ and $1 \mu \mathrm{g} / \mathrm{ml}$, respectively (Nunnally et al., 2019). MIC values for echinocandins among the same isolates ranged from 0.03 to 4 $\mu \mathrm{g} / \mathrm{ml}$ for anidulafungin, 0.03 to $>16 \mu \mathrm{g} / \mathrm{ml}$ for caspofungin, and 0.008 to $>16 \mu \mathrm{g} / \mathrm{ml}$ for micafungin. Another study assessing 87 C. glabrata isolates showed mean MIC distributions ranging from 0.31 to 8 and 0.03 to $2.52 \mu \mathrm{g} / \mathrm{ml}$ for ibrexafungerp and micafungin, respectively (Jimenez-Ortigosa et al., 2017).

In 39 wild-type C. glabrata isolates, MIC values of ibrexafungerp ranged from 0.25 to $1 \mu \mathrm{g} / \mathrm{ml}$ with a modal MIC of $0.5 \mu \mathrm{g} / \mathrm{ml}$ (Pfaller et al., 2017). In the same isolates, modal MIC values of anidulafungin, caspofungin, and micafungin were $0.06,0.06$, and $0.015 \mu \mathrm{g} / \mathrm{ml}$, respectively. In 28 echinocandin resistant isolates, ibrexafungerp MIC values ranged from 0.12 to $16 \mu \mathrm{g} / \mathrm{ml}$ with a modal MIC value of $1 \mu \mathrm{g} / \mathrm{ml}$. In comparison, anidulafungin, caspofungin, and micafungin showed MIC ranges of 0.015 to $4,0.03$ to 16 , and $\leq 0.008$ to $4 \mu \mathrm{g} / \mathrm{ml}$, respectively, with modal MIC values of $1,0.5$, and $0.06 \mu \mathrm{g} / \mathrm{ml}$, respectively.

In two independent studies that evaluated over 400 clinical Candida species isolates collected between 2005 and 2015, in vitro MIC data was gathered to determine the activity of ibrexafungerp against multi-drug resistant C. glabrata strains with resistance to both echinocandins and fluconazole. A total of 17 C. glabrata isolates were resistant to echinocandins and fluconazole. MIC values for ibrexafungerp, caspofungin, micafungin, and fluconazole were $\geq 0.5, \geq 2, \geq 0.25$, and $\geq 64 \mu \mathrm{g} / \mathrm{ml}$, respectively. Ibrexafungerp was found to be active in vitro against $12 / 17$ (71\%) of C. glabrata resistant strains tested (Borroto-Esoda et al., 2017a).

A collection of 72 C. glabrata strains from hospitals throughout the United Kingdom between January 2015 and March 2016 were utilized to assess the in vitro activity of ibrexafungerp. Against echinocandin resistant C. glabrata isolates, $\mathrm{MIC}_{50}$ and $\mathrm{MIC}_{90}$ values were both $1 \mu \mathrm{g} / \mathrm{ml}$ for ibrexafungerp and amphotericin B. Anidulafungin and caspofungin had $\mathrm{MIC}_{50}$ and $\mathrm{MIC}_{90}$ values of 0.25 and $1 \mu \mathrm{g} / \mathrm{ml}$, respectively. The $\mathrm{MIC}_{50}$ and $\mathrm{MIC}_{90}$ of micafungin were 0.06 and $0.25 \mu \mathrm{g} / \mathrm{ml}$, respectively. For fluconazole, the $\mathrm{MIC}_{50}$ and $\mathrm{MIC}_{90}$ values were 2 and $32 \mu \mathrm{g} / \mathrm{ml}$, respectively. Additionally, voriconazole and posaconazole $\mathrm{MIC}_{50}$ values were 0.125 and $0.5 \mu \mathrm{g} / \mathrm{ml}$, respectively, while they both had a $\mathrm{MIC}_{90}$ value of 1 $\mu \mathrm{g} / \mathrm{ml}$ (Table 1) (Borroto-Esoda et al., 2017b).

A compilation across five independent studies was performed to identify the in vitro MIC data for ibrexafungerp against
TABLE 1 | MIC values $(\mu \mathrm{g} / \mathrm{ml})$ of ibrexafungerp and comparators against 72 echinocandin-resistant C. glabrata isolates (Lass-Florl, 2009).

\begin{tabular}{lcccccccc} 
Antifungal & IBX & AMB & ANID & CASP & MICA & FLZ & VORI & POSA \\
\hline MIC $_{50}$ & 1 & 1 & 0.25 & 0.25 & 0.06 & 2 & 0.125 & 0.5 \\
MIC $_{90}$ & 1 & 1 & 1 & 1 & 0.25 & 32 & 1 & 1
\end{tabular}

C. glabrata isolates with $f k s$ mutations (30 fks 1 and $49 f k s 2$ ). Ibrexafungerp showed activity against $78 \%$ of $C$. glabrata isolates with $f k s$ mutations. Additionally, ibrexafungerp demonstrated greater in vitro activity compared to micafungin and caspofungin against $f k s$ mutated C. glabrata strains. The $\mathrm{MIC}_{50}$ of ibrexafungerp against $f k s$ mutant $C$. glabrata ranged from 0.5 to $1 \mu \mathrm{g} / \mathrm{ml}, \mathrm{MIC}_{50}$ of caspofungin ranged from 0.5 to $4 \mu \mathrm{g} / \mathrm{ml}$, and micafungin ranged from 0.125 to $0.25 \mu \mathrm{g} / \mathrm{ml}$ (Barat et al., 2018).

\section{In Vitro Activity at Different pH Levels}

Larkin et al. demonstrated the efficacy of ibrexafungerp in lower $\mathrm{pH}$ environments by evaluating the activity of the drug against Candida species isolated from patients with vulvovaginitis (Larkin et al., 2019). The in vitro activity of ibrexafungerp was shown to be enhanced in lower $\mathrm{pH}$ environments. Against ten $C$. glabrata isolates, the $\mathrm{MIC}_{50}$ values of ibrexafungerp tested at $\mathrm{pH}$ levels of $7.0,5.72$, and 4.5 were $1,0.5$, and $0.063 \mu \mathrm{g} / \mathrm{ml}$, respectively. In comparison, the $\mathrm{MIC}_{50}$ value of micafungin remained the same regardless of $\mathrm{pH}(0.25 \mu \mathrm{g} / \mathrm{ml})$, while the $\mathrm{MIC}_{50}$ values of fluconazole at $\mathrm{pH}$ levels of 7.0, 5.72, and 4.5 were 1,8 , and $8 \mu \mathrm{g} / \mathrm{ml}$, respectively (Table 2 ).

\section{Effect Against Biofilms}

In a study performed in Madrid, Spain, the antifungal activity of ibrexafungerp, micafungin, and fluconazole was evaluated against planktonic and sessile Candida albicans and nonalbicans; including 31 C. glabrata (Marcos-Zambrano et al., 2017). Antifungal susceptibility testing was performed using the Clinical and Laboratory Standards Institute (CLSI) M27A3 and EUCAST EDef 7.3 microdilution broth procedures (Clinical, Institute LS, 2008; Arendrup et al., 2012). The $\mathrm{MIC}_{50}$ and $\mathrm{MIC}_{90}$ values (defined as the concentration that inhibit $50 \%$ and $90 \%$ of isolates tested, respectively) of ibrexafungerp compared to untreated control were 0.125 and $0.25 \mu \mathrm{g} / \mathrm{ml}$, and 0.5 and $1 \mu \mathrm{g} / \mathrm{ml}$ for CLSI and EUCAST, respectively. In contrast, the $\mathrm{MIC}_{50}$ and $\mathrm{MIC}_{90}$ of fluconazole were 2 and $4 \mu \mathrm{g} / \mathrm{ml}$, and 16 and $32 \mu \mathrm{g} / \mathrm{ml}$ CLSI and EUCAST, respectively, while the $\mathrm{MIC}_{50}$ and $\mathrm{MIC}_{90}$ of micafungin were $\leq 0.007$ and $\leq 0.015 \mu \mathrm{g} / \mathrm{ml}$, and 0.125 and $1 \mu \mathrm{g} / \mathrm{ml} \mathrm{CLSI}$ and EUCAST, respectively (Table 3).

In the same study, sessile MICs were assessed by $50 \%$ and $80 \%$ reduction in the metabolic activity of the biofilm following

TABLE 2 | In vitro activity of ibrexafungerp and comparators in lower pH environments (Dodds Ashley et al., 2012).

\begin{tabular}{lccccccccc}
\hline Antifungal & \multicolumn{3}{c}{ Ibrexafungerp } & \multicolumn{3}{c}{ Micafungin } & \multicolumn{3}{c}{ Fluconazole } \\
\hline $\mathrm{PH}$ Level & $\mathbf{7 . 0}$ & $\mathbf{5 . 7 2}$ & $\mathbf{4 . 5}$ & $\mathbf{7 . 0}$ & $\mathbf{5 . 7 2}$ & $\mathbf{4 . 5}$ & $\mathbf{7 . 0}$ & $\mathbf{5 . 7 2}$ & $\mathbf{4 . 5}$ \\
$\mathrm{MIC}_{50}(\mu \mathrm{g} / \mathrm{ml})$ & 1 & 0.5 & 0.063 & 0.25 & 0.25 & 0.25 & 1 & 8 & 8
\end{tabular}


TABLE 3 | MIC values $(\mu \mathrm{g} / \mathrm{ml})$ of ibrexafungerp and comparators against $31 \mathrm{C}$ glabrata isolates using two microdilution broth procedures, the clinical and Laboratory Standards Institute (CLSI) M27-A3 and EUCAST EDef 7.3 (Pfaller M., et al., 2012).

\begin{tabular}{lcccccccc}
\hline & \multicolumn{2}{c}{ CLSI M27-A3 } & & & \multicolumn{3}{c}{ EUCAST EDef 7.3 } \\
\cline { 2 - 5 } \cline { 6 - 8 } & Ibrexafungerp & MICA & FLZ & & Ibrexafungerp & MICA & FLZ \\
\hline $\mathrm{MIC}_{50}$ & 0.125 & $\leq 0.007$ & 2 & & 0.25 & $\leq 0.015$ & 4 \\
$\mathrm{MIC}_{90}$ & 0.5 & 0.125 & 16 & & 1 & 1 & 32
\end{tabular}

antifungal treatment $\left(\mathrm{SMIC}_{50}\right.$ and $\left.\mathrm{SMIC}_{80}\right)$ compared to growth control. The $\mathrm{SMIC}_{50}$ and $\mathrm{SMIC}_{80}$ values of ibrexafungerp in its ability to inhibit the growth of $50 \%$ of the 31 C. glabrata isolates tested were both $0.25 \mu \mathrm{g} / \mathrm{ml}$, while the $\mathrm{SMIC}_{50}$ and $\mathrm{SMIC}_{80}$ required to inhibit growth in $90 \%$ of the isolates were 0.25 and 16 $\mu \mathrm{g} / \mathrm{ml}$, respectively. Ibrexafungerp showed higher activity compared to fluconazole, which had a $\mathrm{SMIC}_{50}$ and $\mathrm{SMIC}_{80}$ value of $\geq 256 \mu \mathrm{g} / \mathrm{ml}$ in its ability to inhibit the growth of $50 \%$ and $90 \%$ of C. glabrata isolates. Micafungin showed similar activity to ibrexafungerp, with $\mathrm{SMIC}_{50}$ and $\mathrm{SMIC}_{80}$ values of $\leq 0.015 \mu \mathrm{g} / \mathrm{ml}$ when inhibiting $50 \%$ of C. glabrata isolates, and $\mathrm{SMIC}_{50}$ and $\mathrm{SMIC}_{80}$ values of 0.25 and $2 \mu \mathrm{g} / \mathrm{ml}$, respectively, when inhibiting $90 \%$ of isolates (Table 4) (Marcos-Zambrano et al., 2017).

\section{Activity of Ibrexafungerp In Vivo}

Ibrexafungerp has shown efficacy in in vivo models (Lepak et al., 2015; Wiederhold et al., 2018). Wiederhold et al. assessed the efficacy of ibrexafungerp administered orally against both wildtype and echinocandin-resistant C. glabrata infections in a neutropenic mouse model (Table 5). Mice were treated with either ibrexafungerp (including 30 or $40 \mathrm{mg} / \mathrm{kg}$ ), caspofungin (1 $\mathrm{mg} / \mathrm{kg}$ ), or placebo for seven days. Ibrexafungerp at $30 \mathrm{mg} / \mathrm{kg}$

TABLE 4 | Sessile MICs at 50\% and 80\% reduction in the metabolic activity of the biofilm of 31 C. glabrata isolates (SMIC50 and SMIC80) following antifungal treatment compared to growth control (Pfaller M. et al., 2012).

\begin{tabular}{|c|c|c|c|c|c|c|}
\hline & \multicolumn{3}{|c|}{$\mathrm{SMIC}_{50}$} & \multicolumn{3}{|c|}{$\mathrm{SMIC}_{80}$} \\
\hline & Ibrexafungerp & MICA & FLZ & Ibrexafungerp & MICA & FLZ \\
\hline $\begin{array}{l}50 \% \text { of } \\
\text { Isolates }\end{array}$ & 0.25 & $\leq 0.015$ & $\geq 256$ & 0.25 & $\leq 0.015$ & $\geq 256$ \\
\hline $90 \%$ of & 0.25 & 0.25 & $\geq 256$ & 16 & 2 & $\geq 256$ \\
\hline
\end{tabular}

significantly reduced the kidneys fungal burden [number of colony forming units (CFUs)/g] in mice infected with both wild-type and echinocandin-resistant isolates compared to placebo $(P<0.01)$. The $40 \mathrm{mg} / \mathrm{kg}$ dose of ibrexafungerp also significantly reduced the kidney fungal burden compared to placebo but only against the echinocandin-resistant isolate $(P<0.01)$. The plasma concentrations of ibrexafungerp were also above the MICs for both wild-type $(0.25 \mu \mathrm{g} / \mathrm{ml})$ and echinocandin-resistant $(1 \mu \mathrm{g} / \mathrm{ml})$ isolates treated with the 30 and $40 \mathrm{mg} / \mathrm{kg}$ doses (combined mean $3.225 \pm 1.023$ and $3.683 \pm$ $0.761 \mu \mathrm{g} / \mathrm{ml}$, respectively) (Wiederhold et al., 2018). In contrast, Ghannoum et al., have shown that mice infected with C. glabrata and treated with ibrexafungerp doses $\leq 30 \mathrm{mg} / \mathrm{kg}$ showed significant reductions in kidney fungal tissue burden in both echinocandin-susceptible and -resistant strains of C. glabrata (Ghannoum et al., 2019).

Another study assessed ibrexafungerp activity against a neutropenic murine model of invasive candidiasis caused by $C$. glabrata. Four C. glabrata isolates were tested against four oral doses, 3.125, 12.5, 50, and $200 \mathrm{mg} / \mathrm{kg}$ of ibrexafungerp salt. Ibrexafungerp demonstrated potent activity in vivo against each isolate with a stasis endpoint achieved at a mean dose of $58.4 \mathrm{mg} /$ $\mathrm{kg}$. MIC range varied 8 -fold (range, 0.03 to $0.25 \mu \mathrm{g} / \mathrm{ml}$ ) achieving a mean tAUC/MIC of 315, and an fAUC/MIC of 0.63 . The drug showed relatively linear pharmacokinetics over the dose range with a maximum concentration of drug in serum $\left(C_{\max }\right)$ increased from $0.04 \mu \mathrm{g} / \mathrm{ml}$ to $2.66 \mu \mathrm{g} / \mathrm{ml}$ over the dose range. A concentration-dependent killing was demonstrated without paradoxical effect over the dose range. Protein binding was $99.8 \%$ and the elimination half-life ranged from $5.9 \mathrm{~h}$ to $8.5 \mathrm{~h}$ in plasma (Lepak et al., 2015).

\section{CLINICAL EFFICACY OF IBREXAFUNGERP}

Another phase 3 open-label, single-arm study showed that oral ibrexafungerp provides favorable therapeutic response when used to treat refractory or intolerant Candida infections, namely $C$. glabrata. Of the 17 patients with invasive candidiasis/candidemia caused by C. glabrata, nine showed complete or partial response $(52.9 \%)$, five had stable disease (29.4\%), and three had their disease progress further $(17.7 \%)$. Ibrexafungerp was also well-tolerated in patients with the most common adverse effect being gastrointestinal (Alexander et al., 2013).

TABLE 5 | Assessment of the efficacy of ibrexafungerp and a comparator against both wild-type and echinocandin-resistant $C$. glabrata infections in a neutropenic mouse model (Lockhart et al., 2012).

\begin{tabular}{|c|c|c|c|c|c|}
\hline \multirow[t]{2}{*}{ Group } & \multirow[t]{2}{*}{ Dose } & \multicolumn{4}{|c|}{ Mean $\log _{10} C F U / g \pm S D$} \\
\hline & & Wild-type & $P$-value vs. Placebo & Echinocandin-resistant & $P$-value vs. Placebo \\
\hline \multicolumn{6}{|l|}{ (Methylcellulose) } \\
\hline \multirow[t]{2}{*}{ Ibrexafungerp } & $30 \mathrm{mg} / \mathrm{kg}$ & $3.57 \pm 0.79$ & $<0.01$ & $2.38 \pm 0.66$ & $<0.01$ \\
\hline & $40 \mathrm{mg} / \mathrm{kg}$ & $4.18 \pm 0.96$ & $P=0.074$ & $2.34 \pm 0.60$ & $<0.01$ \\
\hline Caspofungin & $1 \mathrm{mg} / \mathrm{kg}$ & $2.74 \pm 0.76$ & $P<0.01$ & $3.61 \pm 1.22$ & $>0.05$ \\
\hline
\end{tabular}




\section{SUMMARY AND CONCLUSIONS}

Over the past two decades, C. glabrata has become one of the most common species within the Candida family causing fungal infections. C. glabrata has become a growing concern for mucosal and systemic bloodstream infections and is particularly worrisome because of increasing multidrug resistance to both azoles and echinocandins. Novel antifungal agents in development, such as ibrexafungerp, a first-in-class triterpenoid antifungal, will provide treatment options to address these concerns. Ibrexafungerp allows the advantage of oral administration, unlike prior echinocandins, and is generally safe and well-tolerated with gastrointestinal adverse effects most commonly reported. Several studies have shown equal or superior in vitro activity compared to echinocandin antifungal drugs, such as caspofungin and micafungin, against both wildtype and echinocandin-resistant $C$. glabrata isolates. The in vitro activity of ibrexafungerp has also shown to be efficacious in low

\section{REFERENCES}

(2020). A Phase 3, Multicenter, Randomized, Double-Blind, Placebo-Controlled Study to Evaluate the Efficacy and Safety of Oral Ibrexafungerp (SCY-078) Compared to Placebo in Subjects With Recurrent Vulvovaginal Candidiasis (VVC) [Internet]. https://clinicaltrials.gov/ct2/show/record/NCT04029116? term $=$ NCT04029116\&draw=2\&rank $=1$.

(2020). A Multicenter, Randomized, Double-Blind Study to Evaluate the Safety and Efficacy of the Coadministration of SCY-078 With Voriconazole in Patients With Invasive Pulmonary Aspergillosis [Internet]. https://clinicaltrials.gov/ct2/show/ record/NCT03672292?term $=$ NCT03672292\&draw=2\&rank=1.

(2020). Open-Label Study to Evaluate the Efficacy, Safety, Tolerability and Pharmacokinetics of Oral Ibrexafungerp (SCY-078) as an Emergency Use Treatment for Patients With Candidiasis, Including Candidemia, Caused by Candida Auris [Internet]. https://clinicaltrials.gov/ct2/show/record/ NCT03363841 ? cond=NCT03363841 $\&$ draw $=2 \&$ rank $=1$.

(2020). Open-Label Study to Evaluate the Efficacy and Safety of SCY-078 (Ibrexafungerp) in Patients With Fungal Diseases That Are Refractory to or Intolerant of Standard Antifungal Treatment (FURI) [Internet]. https:// clinicaltrials.gov/ct $2 /$ show $/$ record/NCT03059992?term=FURI\&draw= $2 \&$ rank=1.

Ahmad, A., and Khan, A. U. (2009). Prevalence of Candida species and potential risk factors for vulvovaginal candidiasis in Aligarh, India. Eur. J. Obstet. Gynecol. Reprod. Biol. 144 (1), 68-71. doi: 10.1016/j.ejogrb.2008.12.020

Alexander, B. D., Johnson, M. D., Pfeiffer, C. D., Jimenez-Ortigosa, C., Catania, J., Booker, R., et al. (2013). Increasing echinocandin resistance in Candida glabrata: clinical failure correlates with presence of FKS mutations and elevated minimum inhibitory concentrations. Clin. Infect. Dis. 56 (12), 17241732. doi: $10.1093 / \mathrm{cid} / \mathrm{cit} 136$

Anderson, M. R., Klink, K., and Cohrssen, A. (2004). Evaluation of vaginal complaints. JAMA 291 (11), 1368-1379. doi: 10.1001/jama.291.11.1368

Arendrup, M. C., and Patterson, T. F. (2017). Multidrug-Resistant Candida: Epidemiology, Molecular Mechanisms, and Treatment. J. Infect. Dis. 216 (suppl_3), S445-S451. doi: 10.1093/infdis/jix131

Arendrup, M. C., Cuenca-Estrella, M., Lass-Florl, C., Hope, W., and Eucast, A. (2012). EUCAST technical note on the EUCAST definitive document EDef 7.2: method for the determination of broth dilution minimum inhibitory concentrations of antifungal agents for yeasts EDef 7.2 (EUCAST-AFST). Clin. Microbiol. Infect. 18 (7), E246-E247. doi: 10.1111/j.1469-0691. 2012.03880.x

Arendrup, M. C., Jorgensen, K. M., Hare, R. K., and Chowdhary, A. (2020). In Vitro Activity of Ibrexafungerp (SCY-078) against Candida auris Isolates as Determined by EUCAST Methodology and Comparison with Activity against
$\mathrm{pH}$ environments, which suggests its effectiveness in treating vulvovaginitis. Additional preclinical and clinical studies will be required to further confirm the efficacy and safety of ibrexafungerp in treating fungal infections caused by $C$. glabrata and other causes of invasive fungal diseases.

\section{AUTHOR CONTRIBUTIONS}

All authors listed have made a substantial, direct, and intellectual contribution to the work and approved it for publication.

\section{FUNDING}

This work was supported by a grant from the National Institutes of Health (NIH grant \# R21 AI143305-01).
C. albicans and C. glabrata and with the Activities of Six Comparator Agents. Antimicrob. Agents Chemother. 64 (3), e02136-19. doi: 10.1128/AAC.02136-19 Barat, S., Borroto-Esoda, K., and Angulo, D. (Eds.) (2018). Ibrexafungerp (formerly SCY-078) Displays Potent In Vitro Activity Against C. glabrata Isolates with Mutations in fks Genes. (Lisbon, Portugal: European Society of Clinical Microbiology and Infectious Diseases/American Society Microbiology (ESCMID/ASM). Available at: https://d1io3yog0oux5.cloudfront.net/ 98dd714dc33bcf17951bb234b5ca3d63/scynexis/db/385/2867/pdf/POSTER_ Ibrexafungerp+\%28formerly+SCY-078\%29+Displays+Potent+In+Vitro +Activity+Against+C.+Glabrata+Isolates+with+Mutations+in+fks+Genes_ ESCMID+ASM+2018.pdf.

Barat SB-E, A. D. (2020). "Ibrexafungerp demonstrates potent and consistent in vitro activity against $>400$ global Candida auris isolates, including isolates with elevated MIC's to echinocandins," In: The European Society of Clinical Microbiology and Infectious Diseases (ECCMID) Abstract Book. (Paris, France:The European Society of Clinical Microbiology and Infectious Diseases (ECCMID)). pp. 1728. Available at: https://www.escmid.org/ fileadmin/src/media/PDFs/Abstractbook2020_05.05.2020_Part2.pdf.

Bassetti, M., Righi, E., Costa, A., Fasce, R., Molinari, M. P., Rosso, R., et al. (2006). Epidemiological trends in nosocomial candidemia in intensive care. $B M C$ Infect. Dis. 6, 21. doi: 10.1186/1471-2334-6-21

Berkow, E. L., Angulo, D., and Lockhart, S. R. (2017). In Vitro Activity of a Novel Glucan Synthase Inhibitor, SCY-078, against Clinical Isolates of Candida auris. Antimicrob. Agents Chemother. 61 (7), e00435-17. doi: 10.1128/AAC.00435-17

Beyda, N. D., John, J., Kilic, A., Alam, M. J., Lasco, T. M., and Garey, K. W. (2014). FKS mutant Candida glabrata: risk factors and outcomes in patients with candidemia. Clin. Infect. Dis. 59 (6), 819-825. doi: 10.1093/cid/ciu407

Borroto-Esoda, K., Birchall, S., Moore, C. B., and Angulo, D. (Eds.) (2017a). Assessment of the In Vitro Antifungal Activity of SCY-078 Against a Panel of Susceptible and Resistant, Clinical Candida Isolates from Europe. (New Orleans, LA: ASM). Available at: https://www.abstractsonline.com/pp8/\#!/4358/ presentation/6655.

Borroto-Esoda, K., Scorneaux, B., Angulo, D., and Helou, S. (2017b). In vitro Interaction between SCY-078, Echinocandins and Azoles against Susceptible \& Resistant Candida spp. Determined by the Checkerboard Method (Atlanta, GA: American Society of Microbiology). Available at: https://www.abstractsonline. com/pp8/\#!/4358/presentation/6660.

Borst, A., Raimer, M. T., Warnock, D. W., Morrison, C. J., and Arthington-Skaggs, B. A. (2005). Rapid acquisition of stable azole resistance by Candida glabrata isolates obtained before the clinical introduction of fluconazole. Antimicrob. Agents Chemother. 49 (2), 783-787. doi: 10.1128/AAC.49.2.783-787.2005

Carruthers, C., Barat, S., Thomas, P., and Lewis, E. (2018). SCY-078, a novel IV/ oral triterpenoid anti-fungal treatment in development for vulvovaginal 
candidiasis and other severe invasive fungal infections, is not embryo/fetotoxic. Am. J. Obstet. Gynecol. 219 (6), 646-647. doi: 10.1016/j.ajog.2018.10.043

Castanheira, M., Messer, S. A., Rhomberg, P. R., Dietrich, R. R., Jones, R. N., and Pfaller, M. A. (2014). Isavuconazole and nine comparator antifungal susceptibility profiles for common and uncommon Candida species collected in 2012: application of new CLSI clinical breakpoints and epidemiological cutoff values. Mycopathologia 178 (1-2), 1-9. doi: 10.1007/s11046-014-9772-2

Cetin, M., Ocak, S., Gungoren, A., and Hakverdi, A. U. (2007). Distribution of Candida species in women with vulvovaginal symptoms and their association with different ages and contraceptive methods. Scand. J. Infect. Dis. 39 (6-7), 584-588. doi: 10.1080/00365540601148491

Chakrabarti, A., Chatterjee, S. S., Rao, K. L., Zameer, M. M., Shivaprakash, M. R., Singhi, S., et al. (2009). Recent experience with fungaemia: change in species distribution and azole resistance. Scand. J. Infect. Dis. 41 (4), 275-284. doi: $10.1080 / 00365540902777105$

Cho, E. J., Shin, J. H., Kim, S. H., Kim, H. K., Park, J. S., Sung, H., et al. (2015). Emergence of multiple resistance profiles involving azoles, echinocandins and amphotericin B in Candida glabrata isolates from a neutropenia patient with prolonged fungaemia. J. Antimicrob. Chemother. 70 (4), 1268-1270. doi: $10.1093 / \mathrm{jac} / \mathrm{dku} 518$

Clinical, Institute LS (2008). Reference method for broth dilution antifungal susceptibility testing of yeasts (Wayne, PA: Clinical and Laboratory Standards Institute).

Corsello, S., Spinillo, A., Osnengo, G., Penna, C., Guaschino, S., Beltrame, A., et al. (2003). An epidemiological survey of vulvovaginal candidiasis in Italy. Eur. J. Obstet. Gynecol. Reprod. Biol. 110 (1), 66-72. doi: 10.1016/s0301-2115(03) 00096-4

Dan, M., Segal, R., Marder, V., and Leibovitz, A. (2006). Candida colonization of the vagina in elderly residents of a long-term-care hospital. Eur. J. Clin. Microbiol. Infect. Dis. 25 (6), 394-396. doi: 10.1007/s10096-006-0150-y

Davis, M. R., Donnelley, M. A., and Thompson, G. R. (2020). Ibrexafungerp: A novel oral glucan synthase inhibitor. Med. Mycol. 58 (5), 579-592. doi: $10.1093 / \mathrm{mmy} / \mathrm{myz} 083$

Dodds Ashley, E., Drew, R., Johnson, M., Danna, R., Dabrowski, D., Walker, V., et al. (2012). Cost of invasive fungal infections in the era of new diagnostics and expanded treatment options. Pharmacotherapy 32 (10), 890-901. doi: 10.1002/ j.1875-9114.2012.01124

Fan, S. R., Liao, Q. P., Liu, X. P., Liu, Z. H., and Zhang, D. (2008). Vaginal allergic response in women with vulvovaginal candidiasis. Int. J. Gynaecol. Obstet. 101 (1), 27-30. doi: 10.1016/j.ijgo.2007.08.024

Farmakiotis, D., Tarrand, J. J., and Kontoyiannis, D. P. (2014). Drug-resistant Candida glabrata infection in cancer patients. Emerg. Infect. Dis. 20 (11), 18331840. doi: 10.3201/eid2011.140685

Fidel, P. L.Jr., Vazquez, J. A., and Sobel, J. D. (1999). Candida glabrata: review of epidemiology, pathogenesis, and clinical disease with comparison to C. albicans. Clin. Microbiol. Rev. 12 (1), 80-96. doi: 10.1128/CMR.12.1.80

Ghannoum, M. A., and Rice, L. B. (1999). Antifungal agents: mode of action, mechanisms of resistance, and correlation of these mechanisms with bacterial resistance. Clin. Microbiol. Rev. 12 (4), 501-517. doi: 10.1128/CMR.12.4.501

Ghannoum, M., Long, L., Larkin, E. L., Isham, N., Sherif, R., Borroto-Esoda, K., et al. (2018). Evaluation of the Antifungal Activity of the Novel Oral Glucan Synthase Inhibitor SCY-078, Singly and in Combination, for the Treatment of Invasive Aspergillosis. Antimicrob. Agents Chemother. 62 (6), e00244-18. doi: 10.1128/AAC.00244-18

Ghannoum, M., Long, L., Isham, N., Hager, C., Wilson, R., Borroto-Esoda, K., et al. (2019). Activity of a novel 1,3-beta-D-glucan Synthase Inhibitor, Ibrexafungerp (formerly SCY-078), Against Candida glabrata. Antimicrob. Agents Chemother. 63 (12), e01510-19. doi: 10.1128/AAC.01510-19

Ghannoum, M., Arendrup, M. C., Chaturvedi, V. P., Lockhart, S. R., McCormick, T. S., Chaturvedi, S., et al. (2020a). Ibrexafungerp: A Novel Oral Triterpenoid Antifungal in Development for the Treatment of Candida auris Infections. Antibiotics (Basel) 9 (9), 539. doi: 10.3390/antibiotics 9090539

Ghannoum, M., Isham, N., Angulo, D., Borroto-Esoda, K., Barat, S., and Long, L. (2020b). Efficacy of Ibrexafungerp (SCY-078) against Candida auris in an In Vivo Guinea Pig Cutaneous Infection Model. Antimicrob. Agents Chemother. 64 (10), e00854-20. doi: 10.1128/AAC.00854-20

Goswami, R., Dadhwal, V., Tejaswi, S., Datta, K., Paul, A., Haricharan, R. N., et al. (2000). Species-specific prevalence of vaginal candidiasis among patients with diabetes mellitus and its relation to their glycaemic status. J. Infect. 41 (2), 162166. doi: 10.1053/jinf.2000.0723

Grigoriou, O., Baka, S., Makrakis, E., Hassiakos, D., Kapparos, G., and Kouskouni, E. (2006). Prevalence of clinical vaginal candidiasis in a university hospital and possible risk factors. Eur. J. Obstet. Gynecol. Reprod. Biol. 126 (1), 121-125. doi: 10.1016/j.ejogrb.2005.09.015

Healey, K. R., Zhao, Y., Perez, W. B., Lockhart, S. R., Sobel, J. D., Farmakiotis, D., et al. (2016). Prevalent mutator genotype identified in fungal pathogen Candida glabrata promotes multi-drug resistance. Nat. Commun. 7, 11128. doi: $10.1038 /$ ncomms 11128

Heasley, B. H., Pacofsky, G. J., Mamai, A., Liu, H., Nelson, K., Coti, G., et al. (2012). Synthesis and biological evaluation of antifungal derivatives of enfumafungin as orally bioavailable inhibitors of beta-1,3-glucan synthase. Bioorg. Med. Chem. Lett. 22 (22), 6811-6816. doi: 10.1016/j.bmcl.2012.05.031

Holland, J., Young, M. L., Lee, O., and CAC, S. (2003). Vulvovaginal carriage of yeasts other than Candida albicans. Sex. Transm. Infect. 79 (3), 249-250. doi: $10.1136 /$ sti.79.3.249

Jimenez-Ortigosa, C., Paderu, P., Motyl, M. R., and Perlin, D. S. (2014). Enfumafungin derivative MK-3118 shows increased in vitro potency against clinical echinocandin-resistant Candida Species and Aspergillus species isolates. Antimicrob. Agents Chemother. 58 (2), 1248-1251. doi: 10.1128/ AAC.02145-13

Jimenez-Ortigosa, C., Perez, W. B., Angulo, D., Borroto-Esoda, K., and Perlin, D. S. (2017). Novo Acquisition of Resistance to SCY-078 in Candida glabrata Involves FKS Mutations That both Overlap and Are Distinct from Those Conferring Echinocandin Resistance. Antimicrob. Agents Chemother. 61 (9), e00833-17. doi: 10.1128/AAC.00833-17

Kaur, R., Domergue, R., Zupancic, M. L., and Cormack, B. P. (2005). A yeast by any other name: Candida glabrata and its interaction with the host. Curr. Opin. Microbiol. 8 (4), 378-384. doi: 10.1016/j.mib.2005.06.012

Larkin, E., Hager, C., Chandra, J., Mukherjee, P. K., Retuerto, M., Salem, I., et al. (2017). The Emerging Pathogen Candida auris: Growth Phenotype, Virulence Factors, Activity of Antifungals, and Effect of SCY-078, a Novel Glucan Synthesis Inhibitor, on Growth Morphology and Biofilm Formation. Antimicrob. Agents Chemother. 61 (5), e02396-16. doi: 10.1128/AAC.02396-16

Larkin, E. L., Long, L., Isham, N., Borroto-Esoda, K., Barat, S., Angulo, D., et al. (2019). A Novel 1,3-Beta-d-Glucan Inhibitor, Ibrexafungerp (Formerly SCY078), Shows Potent Activity in the Lower $\mathrm{pH}$ Environment of Vulvovaginitis. Antimicrob. Agents Chemother. 63 (5), e02611-18. doi: 10.1128/AAC.02611-18 Lass-Florl, C. (2009). The changing face of epidemiology of invasive fungal disease in Europe. Mycoses 52 (3), 197-205. doi: 10.1111/j.1439-0507.2009.01691.x

Lee, A., Prideaux, B., Zimmerman, M., Carter, C., Barat, S., Angulo, D., et al. (2020). Penetration of Ibrexafungerp (Formerly SCY-078) at the Site of Infection in an Intra-abdominal Candidiasis Mouse Model. Antimicrob. Agents Chemother. 64 (3), e02268-19. doi: 10.1128/AAC.02268-19

Lepak, A. J., Marchillo, K., and Andes, D. R. (2015). Pharmacodynamic target evaluation of a novel oral glucan synthase inhibitor, SCY-078 (MK-3118), using an in vivo murine invasive candidiasis model. Antimicrob. Agents Chemother. 59 (2), 1265-1272. doi: 10.1128/AAC.04445-14

Lim, C. S., Rosli, R., Seow, H. F., and Chong, P. P. (2012). Candida and invasive candidiasis: back to basics. Eur. J. Clin. Microbiol. Infect. Dis. 31 (1), 21-31. doi: 10.1007/s10096-011-1273-3

Lockhart, S. R., Iqbal, N., Cleveland, A. A., Farley, M. M., Harrison, L. H., Bolden, C. B., et al. (2012). Species identification and antifungal susceptibility testing of Candida bloodstream isolates from population-based surveillance studies in two U.S. cities from 2008 to 2011. J. Clin. Microbiol. 50 (11), 3435-3442. doi: 10.1128/JCM.01283-12

Makanjuola, O., Bongomin, F., and Fayemiwo, S. A. (2018). An Update on the Roles of Non-albicans Candida Species in Vulvovaginitis. J. Fungi (Basel) 4 (4), 121. doi: 10.3390/jof 4040121

Marcos-Zambrano, L. J., Gomez-Perosanz, M., Escribano, P., Bouza, E., and Guinea, J. (2017). The novel oral glucan synthase inhibitor SCY-078 shows in vitro activity against sessile and planktonic Candida spp. J. Antimicrob. Chemother. 72 (7), 1969-1976. doi: 10.1093/jac/dkx010

Matsumoto, E., Boyken, L., Tendolkar, S., McDanel, J., Castanheira, M., Pfaller, M., et al. (2014). Candidemia surveillance in Iowa: emergence of echinocandin resistance. Diagn. Microbiol. Infect. Dis. 79 (2), 205-208. doi: 10.1016/ j.diagmicrobio.2014.02.016 
Mohanty, S., Xess, I., Hasan, F., Kapil, A., Mittal, S., and Tolosa, J. E. (2007). Prevalence \& susceptibility to fluconazole of Candida species causing vulvovaginitis. Indian J. Med. Res. 126 (3), 216-219.

Murphy, G., Darpo, B., Marbury, T., Hyman, M., and Angulo, D. (2017). "Lack of an effect of SCY-078 a novel antifungal agent on QTc interval in healthy subjects," In: Abstracts of ASM Microbe, New Orleans, LA, USA, 2017. Abstract 172. Washington, DC, USA: ASM Press. Available at: https://www.tandfonline. com/doi/full/10.1080/13543784.2020.1791820

Nunnally, N. S., Etienne, K. A., Angulo, D., Lockhart, S. R., and Berkow, E. L. (2019). In Vitro Activity of Ibrexafungerp, a Novel Glucan Synthase Inhibitor against Candida glabrata Isolates with FKS Mutations. Antimicrob. Agents Chemother. 63 (11), e01692-19. doi: 10.1128/AAC.01692-19

Okungbowa, F. I., Isikhuemhen, O. S., and Dede, A. P. (2003). The distribution frequency of Candida species in the genitourinary tract among symptomatic individuals in Nigerian cities. Rev. Iberoam. Micol. 20 (2), 60-63.

Paulitsch, A., Weger, W., Ginter-Hanselmayer, G., Marth, E., and Buzina, W. (2006). A 5-year (2000-2004) epidemiological survey of Candida and nonCandida yeast species causing vulvovaginal candidiasis in Graz, Austria. Mycoses 49 (6), 471-475. doi: 10.1111/j.1439-0507.2006.01284.x

Pfaller, M. A., and Diekema, D. J. (2007). Epidemiology of invasive candidiasis: a persistent public health problem. Clin. Microbiol. Rev. 20 (1), 133-163. doi: 10.1128/CMR.00029-06

Pfaller, M. A., Messer, S. A., Rhomberg, P. R., Borroto-Esoda, K., and Castanheira, M. (2017). Differential Activity of the Oral Glucan Synthase Inhibitor SCY-078 against Wild-Type and Echinocandin-Resistant Strains of Candida Species. Antimicrob. Agents Chemother. 61 (8), e00161-17. doi: 10.1128/AAC.00161-17

Pfaller, M., Neofytos, D., Diekema, D., Azie, N., Meier-Kriesche, H. U., Quan, S. P., et al. (2012). Epidemiology and outcomes of candidemia in 3648 patients: data from the Prospective Antifungal Therapy (PATH Alliance(R)) registry, 20042008. Diagn. Microbiol. Infect. Dis. 74 (4), 323-331. doi: 10.1016/ j.diagmicrobio.2012.10.003

Pfaller, M. A., Castanheira, M., Lockhart, S. R., Ahlquist, A. M., Messer, S. A., and Jones, R. N. (2012). Frequency of decreased susceptibility and resistance to echinocandins among fluconazole-resistant bloodstream isolates of Candida glabrata. J. Clin. Microbiol. 50 (4), 1199-1203. doi: 10.1128/JCM.06112-11

Pham, C. D., Iqbal, N., Bolden, C. B., Kuykendall, R. J., Harrison, L. H., Farley, M. M., et al. (2014). Role of FKS Mutations in Candida glabrata: MIC values, echinocandin resistance, and multidrug resistance. Antimicrob. Agents Chemother. 58 (8), 4690-4696. doi: 10.1128/AAC.03255-14

Redding, S. W., Marr, K. A., Kirkpatrick, W. R., Coco, B. J., and Patterson, T. F. (2004). Candida glabrata sepsis secondary to oral colonization in bone marrow transplantation. Med. Mycol. 42 (5), 479-481. doi: 10.1080/13693780410001731574

Richardson, M., and Lass-Florl, C. (2008). Changing epidemiology of systemic fungal infections. Clin. Microbiol. Infect. 14 Suppl 4, 5-24. doi: 10.1111/j.14690691.2008.01978.x

Richter, S. S., Galask, R. P., Messer, S. A., Hollis, R. J., Diekema, D. J., and Pfaller, M. A. (2005). Antifungal susceptibilities of Candida species causing vulvovaginitis and epidemiology of recurrent cases. J. Clin. Microbiol. 43 (5), 2155-2162. doi: 10.1128/JCM.43.5.2155-2162.2005

Sanglard, D., and Coste, A. T. (2016). Activity of Isavuconazole and Other Azoles against Candida Clinical Isolates and Yeast Model Systems with Known Azole Resistance Mechanisms. Antimicrob. Agents Chemother. 60 (1), 229-238. doi: 10.1128/AAC.02157-15

Sanglard, D. (2016). Emerging Threats in Antifungal-Resistant Fungal Pathogens. Front. Med. (Lausanne) 3, 11. doi: 10.3389/fmed.2016.00011

Sardi, J. C. O., Scorzoni, L., Bernardi, T., Fusco-Almeida, A. M., and Mendes Giannini, M. J. S. (2013). Candida species: current epidemiology, pathogenicity, biofilm formation, natural antifungal products and new therapeutic options. J. Med. Microbiol. 62 (Pt 1), 10-24. doi: 10.1099/ jmm.0.045054-0

Schell, W. A., Jones, A. M., Borroto-Esoda, K., and Alexander, B. D. (2017). Antifungal Activity of SCY-078 and Standard Antifungal Agents against 178 Clinical Isolates of Resistant and Susceptible Candida Species. Antimicrob. Agents Chemother. 61 (11), e01102-17. doi: 10.1128/AAC.01102-17
Sobel, J. D. (2007). Vulvovaginal candidosis. Lancet 369 (9577), 1961-1971. doi: 10.1016/S0140-6736(07)60917-9

Sojakova, M., Liptajova, D., Borovsky, M., and Subik, J. (2004). Fluconazole and itraconazole susceptibility of vaginal yeast isolates from Slovakia. Mycopathologia 157 (2), 163-169. doi: 10.1023/b:myco.0000020594.35357.b0

Toda, M., Williams, S. R., Berkow, E. L., Farley, M. M., Harrison, L. H., Bonner, L. et al. (2019). Population-Based Active Surveillance for Culture-Confirmed Candidemia - Four Sites, United States, 2012-2016. MMWR Surveill. Summ. 68 (8), 1-15. doi: 10.15585/mmwr.ss6808al

Trucksis, M., Friedman, E., Taylor, A., Delgado, L., Reynders, T., DeSmet, M., et al. (2009). "A phase I single-rising dose study evaluating the safety, tolerability, pharmacokinetics and pharmacodynamics of an oral akt inhibitor in healthy male volunteers,". In: 100th American Association for Cancer Research annual meeting 2009. Volume 69, Issue 9 Supplement, Denver, CO Cancer Research 69 (9 Supplement): Abstract \#3604. (AACR).

Vale-Silva, L., Ischer, F., Leibundgut-Landmann, S., and Sanglard, D. (2013). Gain-of-function mutations in PDR1, a regulator of antifungal drug resistance in Candida glabrata, control adherence to host cells. Infect. Immun. 81 (5), 1709-1720. doi: 10.1128/IAI.00074-13

Walker, S. S., Xu, Y., Triantafyllou, I., Waldman, M. F., Mendrick, C., Brown, N., et al. (2011). Discovery of a novel class of orally active antifungal beta-1,3-Dglucan synthase inhibitors. Antimicrob. Agents Chemother. 55 (11), 5099-5106. doi: 10.1128/AAC.00432-11

Wiederhold, N. P., Najvar, L. K., Jaramillo, R., Olivo, M., Pizzini, J., Catano, G., et al. (2018). Oral glucan synthase inhibitor SCY-078 is effective in an experimental murine model of invasive candidiasis caused by WT and echinocandin-resistant Candida glabrata. J. Antimicrob. Chemother. 73 (2), 448-451. doi: 10.1093/jac/dkx422

Wring, S. A., Randolph, R., Park, S., Abruzzo, G., Chen, Q., Flattery, A., et al. (2017). Preclinical Pharmacokinetics and Pharmacodynamic Target of SCY078, a First-in-Class Orally Active Antifungal Glucan Synthesis Inhibitor, in Murine Models of Disseminated Candidiasis. Antimicrob. Agents Chemother. 61 (4), e02068-16. doi: 10.1128/AAC.02068-16

Wring, S., Murphy, G., Atiee, G., Corr, C., Hyman, M., Willett, M., et al. (2018). Lack of Impact by SCY-078, a First-in-Class Oral Fungicidal Glucan Synthase Inhibitor, on the Pharmacokinetics of Rosiglitazone, a Substrate for CYP450 2C8, Supports the Low Risk for Clinically Relevant Metabolic Drug-Drug Interactions. J. Clin. Pharmacol. 58 (10), 1305-1313. doi: 10.1002/jcph.1146

Wring, S., Borroto-Esoda, K., Solon, E., and Angulo, D. (2019a). SCY-078, a Novel Fungicidal Agent, Demonstrates Distribution to Tissues Associated with Fungal Infections during Mass Balance Studies with Intravenous and Oral $\left[{ }^{14} \mathrm{C}\right] \mathrm{SCY}-078$ in Albino and Pigmented Rats. Antimicrob. Agents Chemother. 63 (2), e02119-e02118. doi: 10.1128/AAC.02119-18

Wring, S., Murphy, G., Atiee, G., Corr, C., Hyman, M., Willett, M., et al. (2019b). Clinical Pharmacokinetics and Drug-Drug Interaction Potential for Coadministered SCY-078, an Oral Fungicidal Glucan Synthase Inhibitor, and Tacrolimus. Clin. Pharmacol. Drug Dev. 8 (1), 60-69. doi: 10.1002/ cpdd. 588

Conflict of Interest: In compliance with the ICMJE uniform disclosure form, all authors declare the following: financial relationships: $\mathrm{MG}$ declares that he received funding from Pfizer, Scynexis, Inc., Cidara Therapeutics, and Amplyx Pharmaceuticals. Authors KBE and DA were employed by Scynexis, Inc.

The remaining authors declare that the research was conducted in the absence of any commercial or financial relationships that could be construed as a potential conflict of interest.

Copyright $\odot 2021$ Gamal, Chu, McCormick, Borroto-Esoda, Angulo and Ghannoum. This is an open-access article distributed under the terms of the Creative Commons Attribution License (CC BY). The use, distribution or reproduction in other forums is permitted, provided the original author(s) and the copyright owner(s) are credited and that the original publication in this journal is cited, in accordance with accepted academic practice. No use, distribution or reproduction is permitted which does not comply with these terms. 